
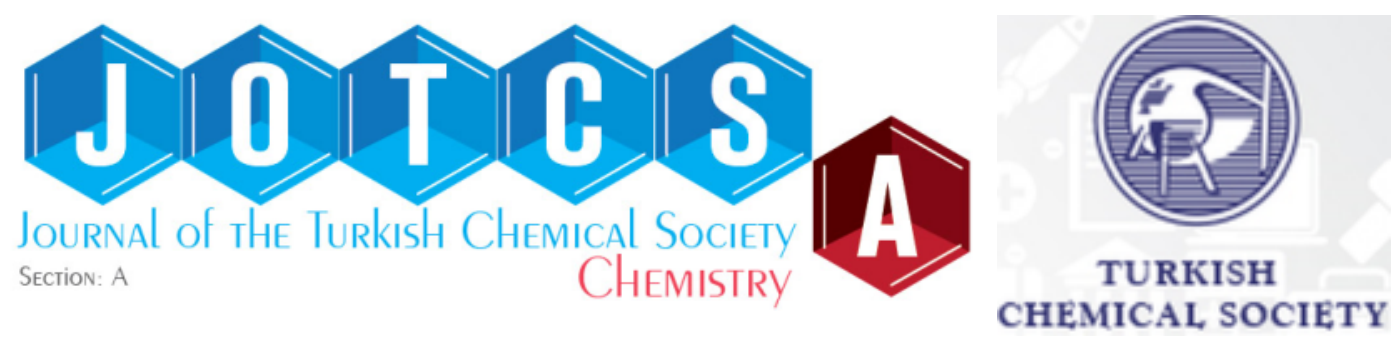

\title{
The Role of the Presence of Aliphatic Alcohols on the Formation of Wormlike Micelle of Anionic-Cationic Surfactants Mixture
}

\author{
Rabah A. Khalil *1 $\triangle$, Liqaa H. Alsamarrai 2 \\ ${ }^{1}$ Department of Chemistry, College of Science, University of Mosul, Mosul, Iraq. \\ 2 Department of Chemistry, College of Education, University of Samarra, Samarra, Iraq.
}

\begin{abstract}
Thermodynamically controlled supramolecular gel or aggregate is commonly resulted from the formation of one-dimensional micelles through specific self-assembling of amphiphilic molecules. This phenomenon is commonly referred to as the formation of worm- or thread-like micelle which is still considered as quite complicated and obscure. This, in spite of the recent physical insight for interpreting this phenomenon, is referred to as the critical intermolecular forces (CIF) of which still needs further support. Therefore, attention by scientists in this field should be given in order to make this transition phenomenon clear. In this work, investigations concerning the effect of addition of aliphatic alcohols with different chain lengths of even carbon numbers (ethanol, 1-butanol, 1-hexanol, 1-octanol and 1-decanol), at different concentrations $(0.002-0.040 \mathrm{M})$, and temperatures on the formation of wormlike micelle for the mixture of anionic sodium dodecylsulfate $(S D S)$ and cationic cetyltrimethylammonium bromide (CTAB) surfactants were carried out. The formation of these one dimensional micelles were practically detected by following the sharp change of physical properties through the presence of a remarkable high viscosity peak. The results indicate there is no linear or systematic relationship between the effects of the presence of these alcohols with their chain lengths. It has been found that the presence of aliphatic alcohols causes a positive effect on the formation of one dimensional micelles. The ability of the transition from spherical to wormlike micelles increases with increasing their concentrations of alcohols except for that of 1-octanol. The obtained achievements were explained in terms of CIF theory which then offered a reasonable support to this theory. It was concluded that alcohols particularly ethanol and 1-octanol can be employed as adjustment reagents for controlling required viscosity of threadlike micelles.
\end{abstract}

Keywords: Wormlike micelles, sodium dodecylsulfate; cetyltrimethylammonium bromide; supramolecular chemistry; soft matter; aliphatic alcohols.

Submitted: July 02, 2021. Accepted: January 19, 2022.

Cite this: Khalil RA, Alsamarrai LH. The Role of the Presence of Aliphatic Alcohols on the Formation of Wormlike Micelle of Anionic-Cationic Surfactants Mixture. JOTCSA. 2022;9(1):275-82.

DOI: https://doi.org/10.18596/jotcsa.961212.

*Corresponding author. E-mail: rakhalil64@yahoo.com.

\section{INTRODUCTION}

There is no doubt concerning with the essential and important applications of surfactants in multi aspects of life. The benefits of use of surface active agents with their traditional spherical micelles are not limited which are increasing day by day (1-4). In special conditions, new version of micelles of one dimensional shape of aggregates which referred as worm- or thread-like micelles are found. The specific characteristics of the rheological behaviors exhibited by the aqueous solution of wormlike micelles promise their employment in very important applications of various aspects (5-15). These properties are released from the thermodynamically controlled supramolecular structure of the self-assembled amphiphilic molecules which are referred to as the living polymers. On the other side, there are only poor efforts concerning the theoretical background of the formation of these one dimensional supramolecular aggregates. Recently, we have developed a new 
physical insight that is called as the Critical Intermolecular Forces (CIF) for interpreting the formation of wormlike micelle (5). This theory was achieved through the investigations of some binary and ternary systems of mixed amphiphilic molecules. The mixture of anionic sodium dodecyl sulfate (SDS) and cationic cetyltrimethylammonium bromide (CTAB) surfactants show a sharp viscosity peak at a weight ratio $80 / 20$ of 3 wt. \% CTAB/SDS. No such remarkable high viscosity peak was found for anionic- and cationic-nonionic (Triton-x 100) nor for ternary mixtures of these surfactants $(5,14)$. The suggested CIF theory proposed that the transition phenomenon from spherical micelle structure to one dimensional is resulted from the combination of three main intermolecular forces. These including electrostatic interactions of polar head groups, dispersion forces between hydrophobic or tail groups and the hydrophobic effect that produced from the excess of formed hydrogen bonds between water molecules (icebergs). The theory (CIF) surprisingly stated that the last effect plays the major role in the formation of one dimensional shape of aggregate as never mentioned in the previous studies. The proposal of CIF interprets the transition process from $3 D$ to $1 D$ as at particular concentrations of molecules, a critical state is created by disturbing the balance of intermolecular interactions which makes the transformation towards 1D energetically more favorable. This theory (CIF) is considered as a helpful tool for interpreting and understanding the transformation to 1D shape of aggregate in addition to the accompanied state of gel is and helpful for predicting a new wormlike systems $(5,14,15)$. Two studies were followed for verification CIF theory $(14,15)$. The first was done through exploring the effect of the presence of benzene ring in the non polar chain of surfactant on the transformation process towards 1D. The results show that the existence of benzene ring in sodium dodecylbenzenesulfonate (SDBS) as a mixture with CTAB comparatively decreases the tendency of forming wormlike micelle which parallel to CIF theory (14). The second study also gives support to CIF through studying the role of surfactant head group in the transition process towards 1D shape of aggregate (15). According to literature, there are several publications concerning the formation of wormlike micelles for gaining more information about the factors affecting the transmission phenomenon towards such type of micelle. No study concerning with effect of alcohol chain length on this phenomenon was mentioned (16-22).

However, as a continuation of our previous investigations, a study of the effect of addition of aliphatic alcohols on the transition process towards 1D seems interesting to us. Indeed, the presence of both of hydrophilic hydroxyl and hydrophobic hydrocarbon groups in these substances may disturb the critical intermolecular forces and even could change the transition mechanism. Therefore, this study could be considered as essential for testing of CIF theory. Therefore, investigations concerning the effect of addition of alcohols with different chain lengths of even carbon numbers (ethanol, 1-butanol, 1-hexanol, 1-octanol and 1decanol), at different concentrations (0.002-0.040 $\mathrm{M}$ ) and temperatures on the formation of wormlike micelle for the mixture of anionic SDS and cationic $C T A B$. Indeed, this study was limited to use only even carbon number of alcohol in order to avoid the effect of zigzag mode which exist in hydrocarbon chains (23).

\section{EXPERIMENTAL SECTION}

The surfactants CTAB $\left(\mathrm{CH}_{3}\left(\mathrm{CH}_{2}\right)_{14} \mathrm{CH}_{2} \mathrm{~N}^{+}\left(\mathrm{CH}_{3}\right)_{3} \mathrm{Br} r^{-}\right)$ and SDS $\left(\mathrm{CH}_{3}\left(\mathrm{CH}_{2}\right)_{10} \mathrm{CH}_{2} \mathrm{OSO}_{3}{ }^{-} \mathrm{Na}^{+}\right.$in addition to all aliphatic alcohols (ethanol, 1-butanol, 1-hexanol, 1octanol and 1-decanol) were used in highly purified form were obtained from VWR Chemicals $\mathrm{BDH}$, Sigma-Aldrich, and Alfa Aesar companies. 3 wt\% aqueous solutions of each surfactant was prepared using freshly distilled water at concentrations of 0.1072 and $0.08486 M$ for CTAB and SDS, respectively. The stock solutions of alcohols were prepared according to their solubility as aqueous surfactants solution of $3 w t \%$ was used as a solvent for non-water soluble alcohols.

Measurements of dynamic viscosity $(\eta)$ were carried out using modified Ostwald apparatus as elaborated in Ref. 5. The temperature was controlled within $\pm 0.1^{\circ} \mathrm{C}$ using water thermostated Hakke NK22. In order to check the reproducibility of the data, all measurements were repeated for at least three times.

\section{RESULTS AND DISCUSSION}

An investigation that deals with the effect of aliphatic alcohols of different chain lengths on the transformation from $3 D$ to $1 D$ shape of aggregate may afford good information about interesting transition. This is due to the fact that the behavior of alcohols differ significantly from each other from molecular interactions point of view. For example, the water solubility of ethanol is very high, while 1butanol is slightly soluble $(7.9 \mathrm{~g} / 100 \mathrm{~mL})$, and 1 hexanol is very slightly soluble $(0.6 \mathrm{~g} / 100 \mathrm{~mL})$, while 1-octanol and 1-decanol are immiscible. As far as the previous study indicates the sharp viscosity peak for the mixture of SDS and CTAB due to presence of worm-like micelle which is lying at weight ratio $80 / 20$ of 3 wt. $\%$ CTAB/SDS (5). Therefore, the first exploration should be about the effect of the presence of alcohols on the position of this peak. The results as illustrated in Figure 1 show there is no effect on the position of this characteristic peak due to presence of these additives. Indeed, such achievement could provide support to the CIF theory as the long length chain of hydrophobic group plays a major role in the formation of wormlike micelles, as that for CTAB is 
exceeding by four $\mathrm{CH}_{2}$ groups in contrast to that of SDS (5).

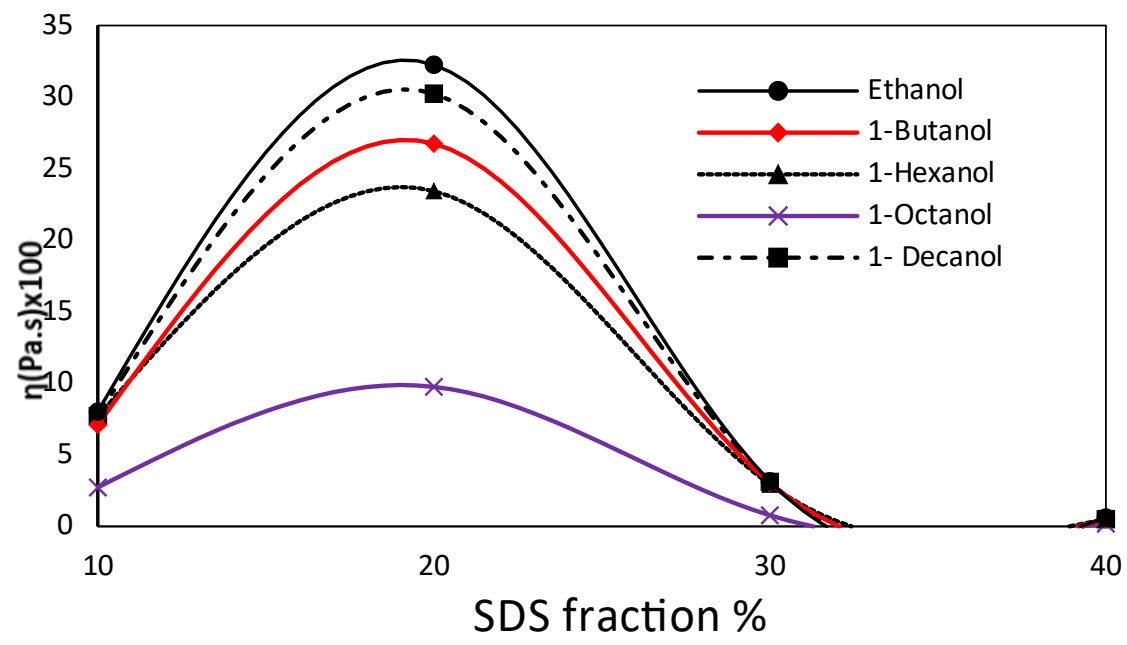

Figure 1: It exhibits that there is no effect due to presence of aliphatic alcohols $(0.01 \mathrm{M})$ on the position of the sharp viscosity peak at a weight ratio $80 / 20$ of $3 \mathrm{wt} \% \mathrm{CTAB} / \mathrm{SDS}$ at $25^{\circ} \mathrm{C}$.

The effect of presence of alcohols at different concentrations of $0.002,0.010,0.020,0.030$, and 0.040 on the sharp viscosity peak were investigated at different temperatures as illustrated in Figures 26 , respectively. In general, these figures show clear enhancement in the ability of the formation of threadlike micelles due to presence of alcohols at different ratios except 1-octanol according to the following sequence:

ethanol>1-decanol> 1-butanol> 1-hexanol > 1octanol

Interestingly, such non-systematic consequence is quite parallel with the principle of CIF theory. However, the significant effect of ethanol may be related to the intermolecular interaction of its hydroxyl group with the head groups of spherical micelle which then reduces the attraction between the two oppositely charged molecules, that is, CTAB and SDS. Then the stability of micelle will be reduced which lead to the transformation phenomenon towards one dimensional micelle. On the other side, the relatively remarkable effect of 1 decanol may be accepted for its long hydrophobic chain of the similar length to that of SDS which may cannot be covered by the hydrophobic core of $3 D$ micelle. Then, 1-decanol may behave as a surfactant through an increase of the hydrophobic effect that is responsible for the transformation towards one dimensional micelle according to CIF theory (5). Another probability of 1-decanol may be released when behaves as a surfactant due to its relatively long hydrophobic chain as involved within the structure of spherical micelle with both CTAB and SDS which then reduces the attraction between head groups leading to the phenomenon of transformation. On the other side, 1-octanol shows a very slight effect towards the transformation phenomenon in comparison with others, even there is a negative effect at relatively high concentration as clearly displayed in Figure 6 . The reason for this comparatively unusual behavior of 1-octanol can be related to the considerable stabilization of the structure of 3D micelle through entering its hydrophobic core. Meanwhile, the behaviors of both 1-butanol and 1-hexanol may be correlated to the hydrophobic effect due to excess in number of $\mathrm{CH}_{2}$ group in contrast to that of both ethanol and 1octanol. In other words, the activity of 1-butanol and 1-hexanol towards the formation of one dimensional micelles can be considered as the mediator between those of ethanol and 1-octanol. 


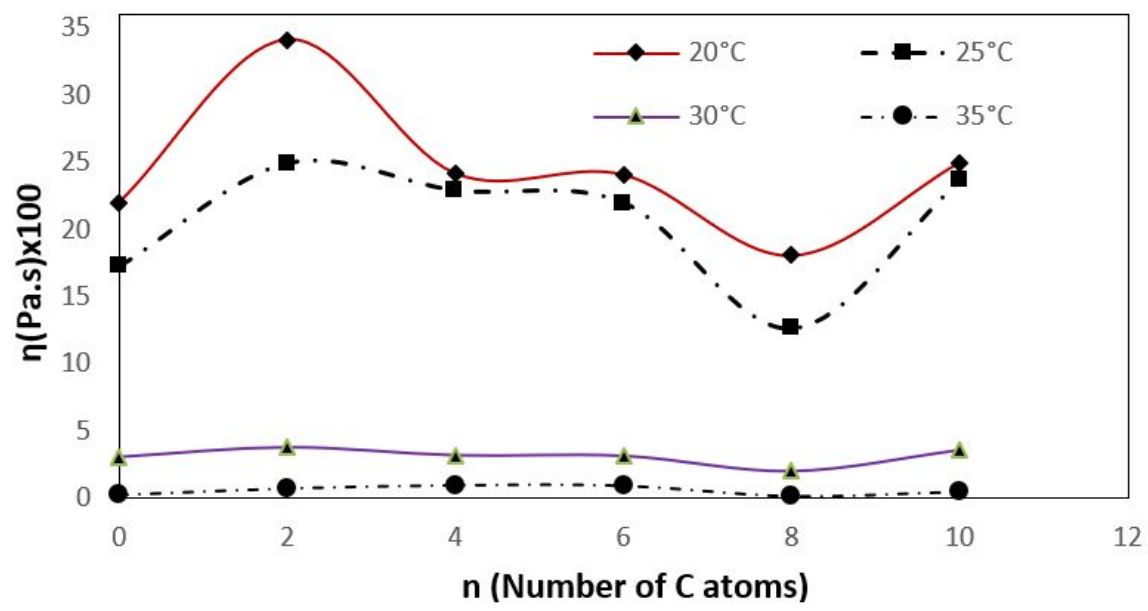

Figure 2: Effect of the presence of aliphatic alcohols (ethanol, 1-butanol, 1-hexanol, 1-octanol and 1decanol) in concentration of $0.002 \mathrm{M}$ on the top viscosity $(n)$ of the wormlike micelle for the mixture of $3 \%$ $w / w C T A B / S D S$ surfactants in water of $80: 20$ ratio at different temperatures.

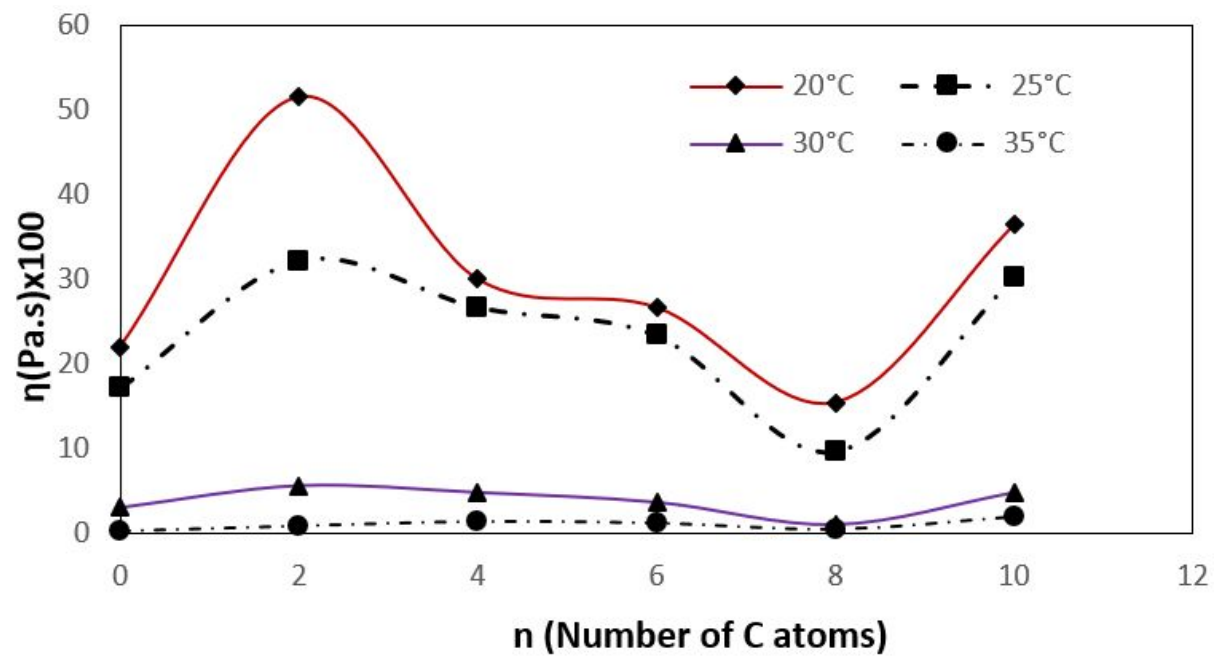

Figure 3: Effect of the presence of aliphatic alcohols (ethanol, 1-butanol, 1-hexanol, 1-octanol, and 1decanol) in concentration of $0.01 \mathrm{M}$ on the top viscosity $(\eta)$ of the wormlike micelle for the mixture of $3 \%$ $\mathrm{w} / \mathrm{w} \mathrm{CTAB} / \mathrm{SDS}$ surfactants in water of $80: 20$ ratio at different temperatures.

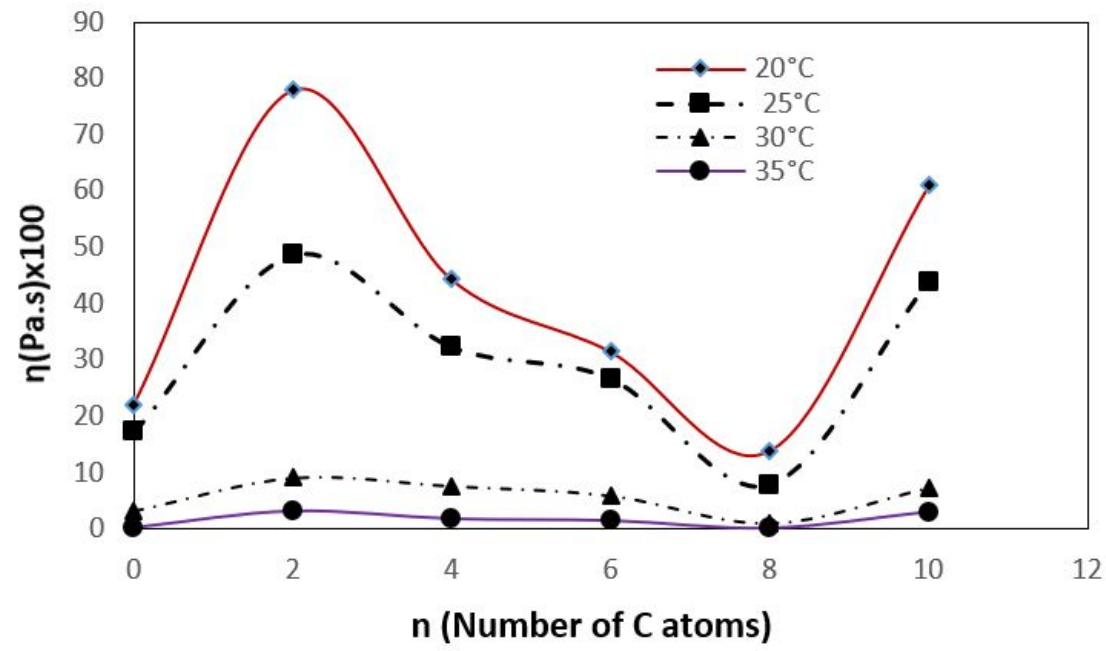

Figure 4: Effect of the presence of aliphatic alcohols (ethanol, 1-butanol, 1-hexanol, 1-octanol, and 1decanol) at a concentration of $0.02 \mathrm{M}$ on the top viscosity $(\eta)$ of the wormlike micelle for the mixture of $3 \%$ $\mathrm{w} / \mathrm{w} \mathrm{CTAB} / \mathrm{SDS}$ surfactants in water of $80: 20$ ratio at different temperatures. 


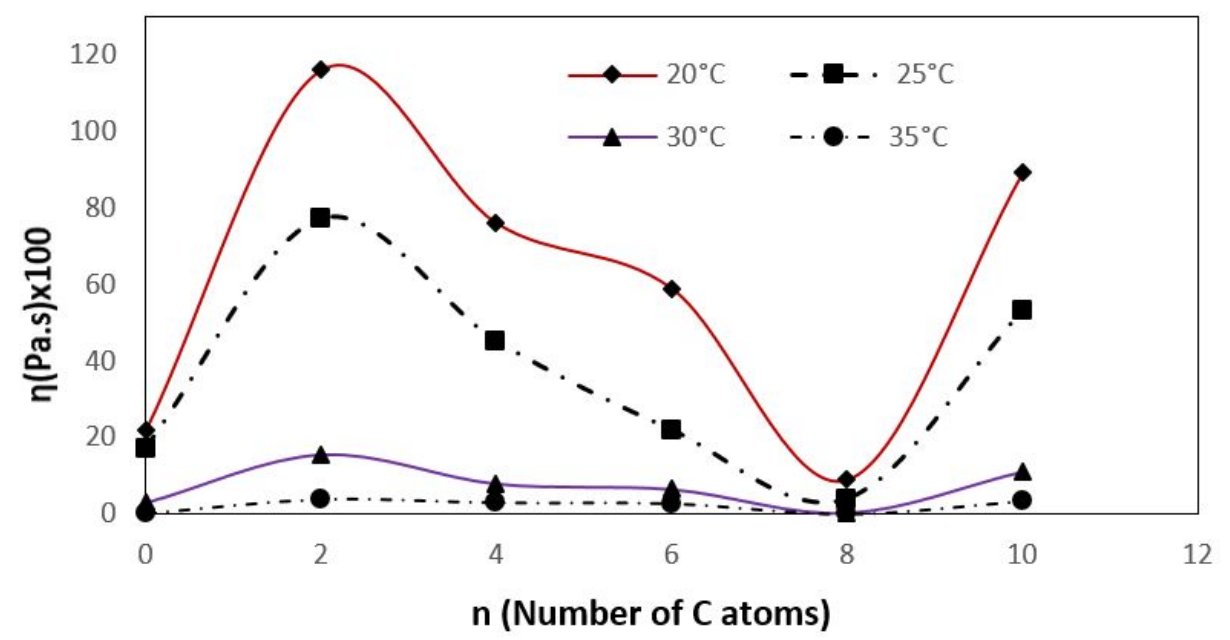

Figure 5: Effect of the presence of aliphatic alcohols (ethanol, 1-butanol, 1-hexanol, 1-octanol, and 1decanol) at a concentration of $0.03 \mathrm{M}$ on the top viscosity $(n)$ of the wormlike micelle for the mixture of $3 \%$ $\mathrm{w} / \mathrm{w}$ CTAB/ SDS surfactants in water of $80: 20$ ratio at different temperatures.

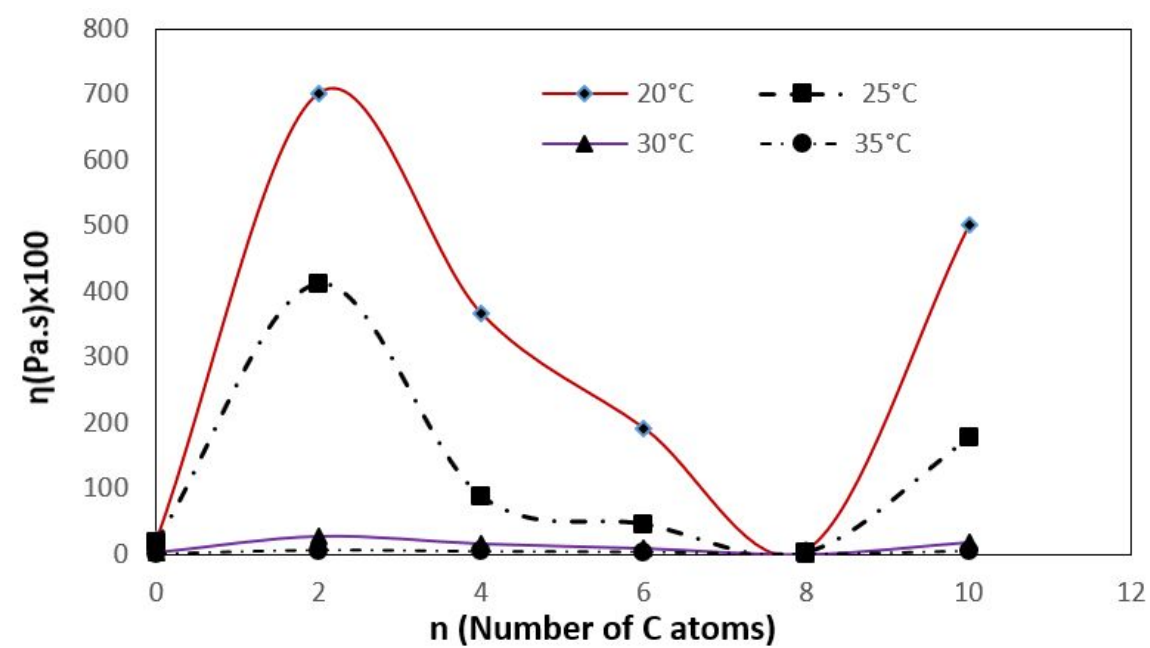

Figure 6: Effect of the presence of aliphatic alcohols (ethanol, 1-butanol, 1-hexanol, 1-octanol, and 1decanol) at a concentration of $0.04 \mathrm{M}$ on the top viscosity $(\eta)$ of the wormlike micelle for the mixture of $3 \%$ $w / w C T A B / S D S$ surfactants in water of $80: 20$ ratio at different temperatures.

Figures 7 and 8 interpret the effect of changing the concentration of aliphatic alcohols on the sharp peak viscosity of $3 \% \mathrm{w} / \mathrm{w}$ CTAB/ SDS surfactants in water of $80: 20$ ratio at 20 and $25{ }^{\circ} \mathrm{C}$, respectively. Indeed, the relationships of those figures indicating that the ability of the formation of wormlike micelles increases with increasing the concentration of alcohols except for that of 1 -octanol. The reason for this can also be related to the same of above discussion for the relationships of Figures 2-6 in terms of CIF theory. 


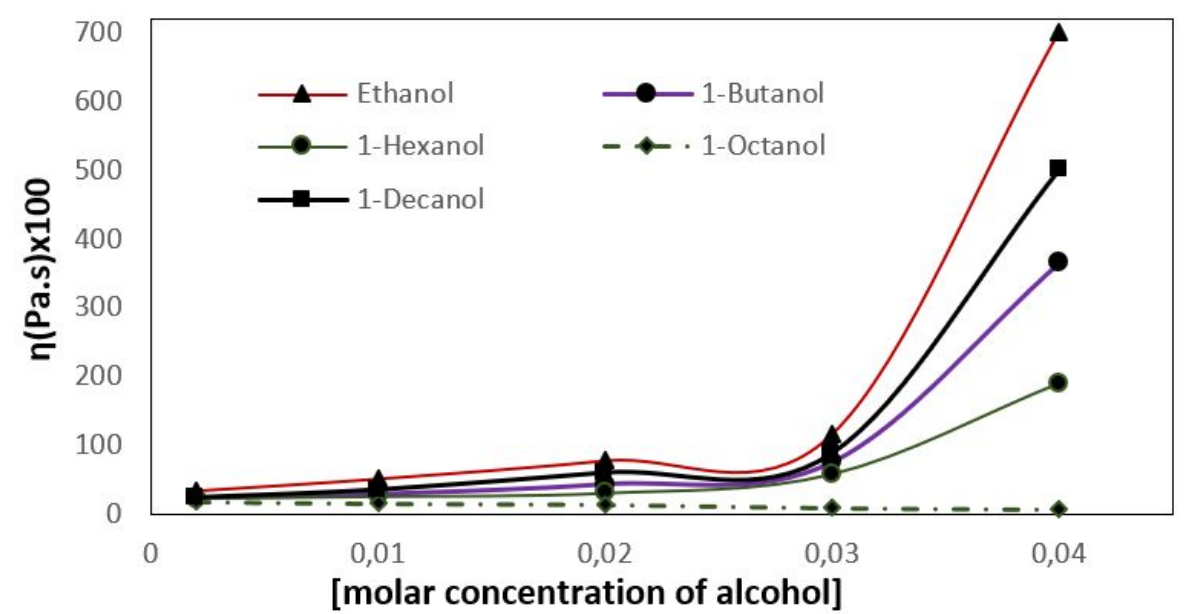

Figure 7: Effect of the presence of aliphatic alcohols (ethanol, 1-butanol, 1-hexanol, 1-octanol and 1decanol) in different concentrations on the top viscosity $(\eta)$ of the wormlike micelle for the mixture of $3 \%$ $\mathrm{w} / \mathrm{w}$ CTAB/ SDS surfactants in water of $80: 20$ ratio at $20{ }^{\circ} \mathrm{C}$ temperature.

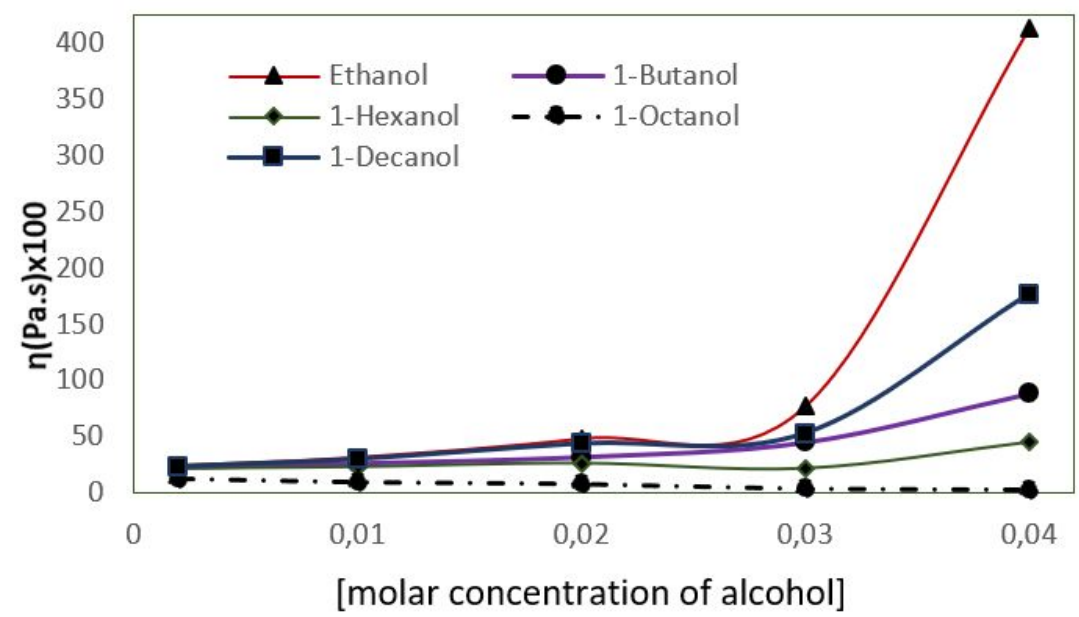

Figure 8: Effect of the presence of aliphatic alcohols (ethanol, 1-butanol, 1-hexanol, 1-octanol, and 1decanol) in different concentrations on the top viscosity $(\eta)$ of the wormlike micelle for the mixture of $3 \%$ $\mathrm{w} / \mathrm{w}$ CTAB/ SDS surfactants in water of $80: 20$ ratio at $25^{\circ} \mathrm{C}$ temperature.

\section{CONCLUSION}

On the basis of the above results, one could conclude that the theory of CIF that coping the transformation occurrence from 3D to 1D shape of aggregate is still the right physical insight of this phenomenon. Hence, the presence of aliphatic alcohols helps for reaching the critical state of intermolecular forces of the formed spherical micelle towards the formation of 1D micelle. While, for 1 octanol the matter is quite different as the presence of 1-octanol satisfies the 3D shape of aggregate which then it may work as inhibitor for reaching the critical state, particularly when exists at relatively high concentration. Finally, the results recommended that alcohols particularly ethanol and 1-octanol can be employed as adjustment reagents for controlling required viscosity of threadlike micelles.

\section{CONFLICT OF INTEREST}

The authors declare that they have no conflict of interest.

\section{ACKNOWLEDGMENTS}

Thanks should go to the University of Mosul for supporting the presented work.

\section{REFERENCES}

1. Mondal MH, Roy A, Malik S, Ghosh A, Saha B. Review on chemically bonded geminis with cationic heads: secondgeneration interfactants. Res Chem Intermed. 2016 Mar;42(3):1913-28. <DOI>.

2. Sar P, Ghosh A, Scarso A, Saha B. Surfactant for better tomorrow: applied aspect of surfactant aggregates from laboratory to industry. Res Chem Intermed. 2019 Dec;45(12):6021-41. <DOI . 
3. Chowdhury S, Rakshit A, Acharjee A, Mahali K, Saha B. Surface phenomenon in micellar media: An excellent controlling factor for oxidation of fatty aldehyde in aqueous medium. Journal of Molecular Liquids. 2020 Jul;310:113224. <DOI $>$.

4. Acharjee A, Rakshit A, Chowdhury S, Saha B. Micelle catalysed conversion of 'on water' reactions into 'in water' one. Journal of Molecular Liquids. 2021 Jan;321:114897. $\leq \mathrm{DOI}>$.

5. Khalil RA, Hammad FJ. Critical Intermolecular Forces: A New Physical Insight for the Formation of Wormlike Micelles. Journal of the Chemical Society of Pakistan. 2014;36(2):211-20.

6. Rodriguez-Abreu C, Shrestha RG, Shrestha LK, Harush E, Regev O. Worm-Like Soft Nanostructures in Nonionic Systems: Principles, Properties and Application as Templates. j nanosci nanotech. 2013 Jul 1;13(7):4497520. $<$ DOI $>$.

7. Parker A, Fieber W. Viscoelasticity of anionic wormlike micelles: effects of ionic strength and small hydrophobic molecules. Soft Matter. 2013;9(4):1203-13. <DOI>

8. Wang Z, Li Y, Dong X-H, Yu X, Guo K, Su H, et al. Giant gemini surfactants based on polystyrene-hydrophilic polyhedral oligomeric silsesquioxane shape amphiphiles: sequential "click" chemistry and solution self-assembly. Chem Sci. 2013;4(3):1345. <DOI>.

9. Dreiss CA. Wormlike micelles: where do we stand? Recent developments, linear rheology and scattering techniques. Soft Matter. 2007;3(8):956. <DOI .

10. Ziserman L, Abezgauz L, Ramon $O$, Raghavan SR, Danino D. Origins of the Viscosity Peak in Wormlike Micellar Solutions. 1. Mixed Catanionic Surfactants. A Cryo-Transmission Electron Microscopy Study. Langmuir. 2009 Sep 15;25(18):10483-9. <DOI>.

11. Li J, Zhao M, Zheng L. Salt-induced wormlike micelles formed by $\mathrm{N}$-alkyl-N-methylpyrrolidinium bromide in aqueous solution. Colloids and Surfaces $A$ : Physicochemical and Engineering Aspects. 2012 Feb;396:16-21. $\leq$ DOI $>$.

12. Dan-Hua X, Jian-Xi Z, Lin L, Yi Y, Xi-Lian W, 1 福州大学 化学化工学院，胶体与界面化学研究所，福州 $350108 ;, 1$ Institute of Colloid and Interface Chemistry, College of Chemistry and Chemical Engineering, Fuzhou University, Fuzhou, Fujian, 350108, P. R. China; et al. A Highly Viscoelastic Anionic Wormlike Micellar System. Acta Physico-Chimica Sinica. 2013;29(07):1534-40. <DOI>.

13. Yu H, Xu Z, Wang D, Chen X, Zhang Z, Yin Q, et al. Intracellular $\mathrm{pH}$-activated PEG-b-PDPA wormlike micelles for hydrophobic drug delivery. Polym Chem. 2013;4(19):5052. <DOI>.

14. Khalil RA, Saadoon FA. Effect of presence of benzene ring in surfactant hydrophobic chain on the transformation towards one dimensional aggregate. Journal of Saudi Chemical Society. 2015 Jul;19(4):423-8. <DOI>.
15. Khalil RA, Alsamarrai LH. The role of surfactants head group in the formation of self-assembled supramolecular aggregate. Arab J Phys Chem. 2015;2(1):7-13.

16. Fieber W, Scheklaukov A, Kunz W, Pleines M, Benczédi $D$, Zemb T. Towards a general understanding of the effects of hydrophobic additives on the viscosity of surfactant solutions. Journal of Molecular Liquids. 2021 May;329:115523. <DOI>.

17. Tan G, Zou W, Weaver M, Larson RG. Determining threadlike micelle lengths from rheometry. Journal of Rheology. 2021 Jan;65(1):59-71. <DOI>.

18. Pandya N, Rajput G, Janni DS, Subramanyam G, Ray $D$, Aswal $V$, et al. SLES/CMEA mixed surfactant system: Effect of electrolyte on interfacial behavior and microstructures in aqueous media. Journal of Molecular Liquids. 2021 Mar;325:115096. <DOI $>$.

19. Mehringer J, Hofmann E, Touraud D, Koltzenburg S, Kellermeier M, Kunz W. Salting-in and salting-out effects of short amphiphilic molecules: a balance between specific ion effects and hydrophobicity. Phys Chem Chem Phys. 2021;23(2):1381-91. <DOI>.

20. Khalil RA, Alsamarrai LH. Study The Effect Of Ethanol On The Formation Of The Wormlike Micelles For A Mixture Of Ionic Surfactants. Ratio. 2015;10(90):4344-89.

21. Shibaev AV, Aleshina AL, Arkharova NA, Orekhov AS, Kuklin AI, Philippova OE. Disruption of Cationic/Anionic Viscoelastic Surfactant Micellar Networks by Hydrocarbon as a Basis of Enhanced Fracturing Fluids Clean-Up. Nanomaterials. 2020 Nov 27;10(12):2353. <DOI>.

22. Moitzi C, Freiberger N, Glatter O. Viscoelastic Wormlike Micellar Solutions Made from Nonionic Surfactants: Structural Investigations by SANS and DLS. J Phys Chem B. 2005 Aug 1;109(33):16161-8. <DOI $>$.

23. Khalil $R$, Zarari $A$. Theoretical investigations for the behavior of hydrotropes in aqueous solution. JOTCSA. 2015;2(4):42-52. $\leq$ URL $>$. 
